\title{
Membership status of TWA22AB
}

\author{
Ramachrisna Teixeira, ${ }^{1}$ Christine Ducourant, ${ }^{2}$ Gael Chauvin,${ }^{3}$ \\ Alberto G. O. Krone-Martins, ${ }^{1}$ Mickael Bonnefoy ${ }^{3}$ and Inseok Song ${ }^{4}$ \\ ${ }^{1}$ Instituto de Astronomia, Geofísica e Ciências Atmosféricas, Universidade de São Paulo, \\ Rua do Matão 1226, Cidade Universitária, São Paulo, Brazil \\ email: teixeira@astro.iag.usp.br \\ ${ }^{2}$ Laboratoire d'Astrophysique de Bordeaux, Université de Bordeaux I, France \\ ${ }^{3}$ Laboratoire d'Astrophysique, Observatoire de Grenoble, France \\ ${ }^{4}$ Department of Physics \& Astronomy, University of Georgia, GA USA
}

\begin{abstract}
Using the ESO NTT/SUSI2 telescope, we observed TWA22AB during five different observing runs over 1.2 years to measure its trigonometric parallax and proper motion. HARPS at the ESO 3.6m telescope was also used to measure the system's radial velocity over 2 years. Based on trigonometric-parallax, proper-motion and radial-velocity measurements, we re-analyzed the membership of TWA22AB of the young, nearby associations TW Hydrae, $\beta$ Pictoris and Tucana-Horologium.
\end{abstract}

Keywords. stars: distances, open clusters and associations: general, stars: individual (TWA22AB), binaries: general

\section{Introduction}

TWA22AB was initially regarded as a member (Song et al. 2003) of the TW Hydrae association (TWA; Kastner et al. 1997). In addition to being one of the youngest $(\approx$ $8 \mathrm{Myr})$ and nearest $(\approx 20 \mathrm{pc})$ stars to Earth, TWA22AB has proved to be very interesting once it was resolved as a tight, very-low-mass binary (Bonnefoy et al. 2009). This binary can serve as a very useful dynamical calibrator for pre-main-sequence evolutionary models (Bonnefoy et al. 2009). However, its membership of the TWA has been questioned recently (Mamajek 2005) due to a lack of accurate kinematic measurements.

Based on trigonometric-parallax, proper-motion and radial-velocity measurements, we aim here to re-analyze the membership of TWA22 of the young, nearby associations TWA, $\beta$ Pictoris ( $\beta$ Pic; Barrado y Navascués et al. 1999) and Tucana-Horologium (Tuc-Hor; Zuckerman \& Webb 2000; Torres et al. 2000).

We report our analysis based on several complementary criteria, resulting in a greater chance of TWA22AB being a member of $\beta$ Pic without excluding the possibility to be a member of the TWA because of large uncertainties in the data of its members. In any case, the probability that it belongs to one of the young and nearby known associations is low (Teixeira et al. 2009).

\section{Astrometric parameters for TWA22AB and radial velocity}

Using the ESO NTT/SUSI2 telescope, we observed TWA22AB during five different observing runs over 1.2 years to measure its trigonometric parallax and proper motion. This is part of a larger project to measure trigonometric parallaxes and proper motions of most known TWA members at submilliarcsec level (Ducourant et al. 2008). HARPS at the ESO 3.6m telescope was also used to measure the system's radial velocity over 2 years. 
Table 1. Astrometric parameters and radial velocity for TWA22AB.

\begin{tabular}{|c|c|c|c|c|c|c|}
\hline $\begin{array}{c}\mathrm{RA} \\
\mathrm{h} \mathrm{m} \mathrm{s}\end{array}$ & $\begin{array}{l}\text { Dec } \\
0,111\end{array}$ & $\begin{array}{c}\pi \\
\operatorname{mas}\end{array}$ & $\begin{array}{c}d \\
\text { pc }\end{array}$ & $\begin{array}{l}\mu_{\alpha} \cos (\delta) \\
\operatorname{mas} \mathrm{yr}^{-1}\end{array}$ & $\begin{array}{c}\mu_{\delta} \\
\text { mas yr }^{-1}\end{array}$ & $\begin{array}{c}V_{\mathrm{rad}} \\
\mathrm{km} \mathrm{s}^{-1}\end{array}$ \\
\hline 101726.79 & -535426.5 & $57.0 \pm 0.7$ & $17.5 \pm 0.2$ & $-175.8 \pm 0.8$ & $-21.3 \pm 0.8$ & $14.8 \pm 2.1$ \\
\hline
\end{tabular}

\section{Membership analysis}

To test the membership of TWA22AB, our first approach is to statistically compare its Galactic space motion with the mean $U V W$ values for TWA, $\beta$ Pic, and Tuc-Hor members. Only stars with known trigonometric parallaxes were considered. For TWA, our sample set includes five members. For $\beta$ Pic, we used 26 and for Tuc-Hor 18 members (Teixeira et al. 2009).

The calculated mean space motions of TWA, $\beta$ Pic and Tuc-Hor are reported in the table below, together with the spatial heliocentric coordinates and velocities for TWA22AB. The mean values derived here for the associations are in good agreement with published values.

Table 2. Heliocentric coordinates $(X, Y, Z)$ and Galactic velocities $(U, V, W)$ of TWA22 AB, together with mean values for TWA, $\beta$ Pic and Tuc-Hor. Positive $X(U)$ points to the Galactic center, $Y(V)$ is positive in the direction of Galactic rotation and $Z(W)$ is positive towards the north Galactic pole.

\begin{tabular}{|c|c|c|c|c|c|c|c|c|}
\hline \multirow[t]{2}{*}{ Name } & $X$ & $Y$ & $Z$ & $U$ & $V$ & $W$ & \multirow{2}{*}{$\begin{array}{l}\text { Age } \\
\text { Myr }\end{array}$} & \multirow{2}{*}{$\begin{array}{c}d \\
\text { pc }\end{array}$} \\
\hline & \multicolumn{3}{|c|}{$\mathrm{pc}$} & \multicolumn{3}{|c|}{$\mathrm{km} \mathrm{s}^{-1}$} & & \\
\hline TWA 22 & $3.5 \pm 0.2$ & $-17.2 \pm 0.2$ & $0.7 \pm 0.2$ & $-8.0 \pm 0.4$ & $-17.1 \pm 2.1$ & $-9.0 \pm 0.2$ & $\leqslant 10(1)$ & $17.5 \pm 0.2$ \\
\hline TWA & $14.4 \pm 8.7$ & $-46.9 \pm 5.3$ & $22.5 \pm 2.5$ & $-10.0 \pm 2.0$ & $-17.6 \pm 1.4$ & $-4.8 \pm 1.1$ & $8(2)$ & $55 \pm 7$ \\
\hline$\beta \mathrm{Pic}$ & $9.2 \pm 28.9$ & $-7.5 \pm 13.0$ & $-13.2 \pm 6.5$ & $-10.9 \pm 1.9$ & $-16.1 \pm 1.0$ & $-9.0 \pm 1.2$ & $12(3)$ & $34 \pm 14$ \\
\hline Tuc-Hor & $14.1 \pm 17.7$ & $-19.4 \pm 8.0$ & $-34.9 \pm 3.5$ & $-9.5 \pm 1.7$ & $-20.6 \pm 1.7$ & $-0.6 \pm 2.6$ & $27(4)$ & $46 \pm 5$ \\
\hline
\end{tabular}

To test the membership of TWA22AB of TWA, $\beta$ Pic and Tuc-Hor based on its space motion, we applied a $\chi^{2}$ test with three degrees of freedom, using their spacemotion measurements. We find that the probabilities that TWA22AB's space motion is compatible with the mean space motion of $\beta \mathrm{Pic}$, TWA and Tuc-Hor are 50, 1 and $0.5 \%$, respectively. An alternative approach is to perform a $k-\mathrm{NN}$ analysis in $U V W$ space, where we have computed the distance of TWA22AB to all members of these associations. Among the $k$ nearest neighbours to TWA22AB, the fraction of members for a given association gives the membership probability to that group. This $k-\mathrm{NN}$ analysis corroborates that TWA22AB is more likely a member of $\beta$ Pic than of TWA or Tuc-Hor. Both calculations tend to reject TWA and Tuc-Hor as a host association for TWA22AB.

Another approach is to count the number of TWA, $\beta$ Pic and Tuc-Hor members (or 'neighbours') within a sphere of fixed radius in $U V W$ space, centered on TWA22AB's value and on each individual member of these associations. Radii are selected proportional to the velocity dispersions of the associations. We found that the $U V W$ spatial densities of TWA and Tuc-Hor members around TWA22AB are significantly smaller than the average for both associations. On the other hand, the $U V W$ spatial density of $\beta$ Pic members around TWA22AB is similar to the average density in $\beta$ Pic, as shown in Figure 1.

Finally, another way to test the membership of TWA22AB of TWA, $\beta$ Pic and Tuc-Hor is to use the trace-back technique to compare the Galactic space position of TWA22 AB 


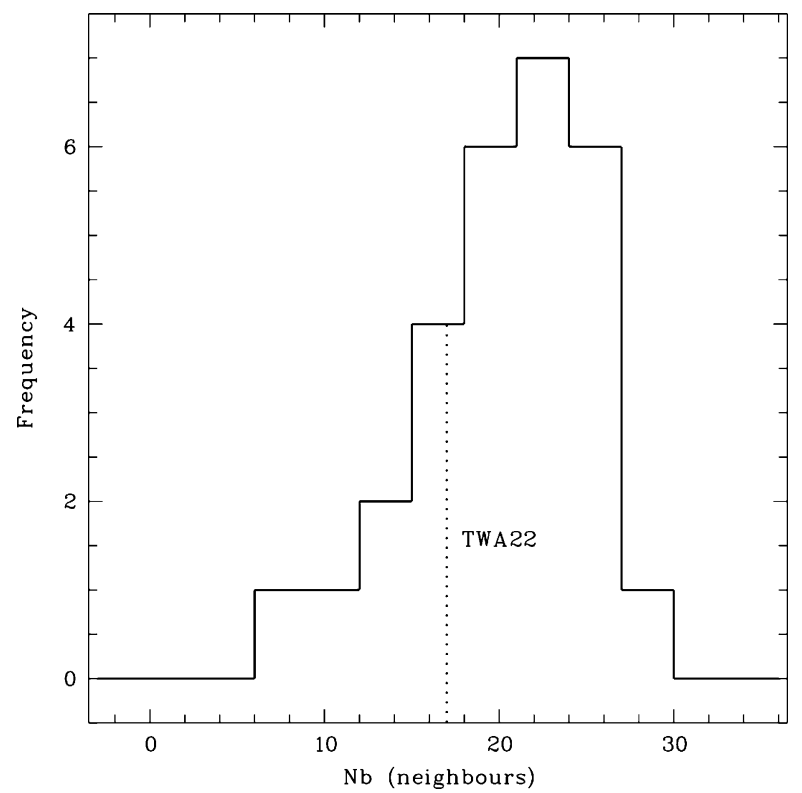

Figure 1. Number of $\beta$ Pic members in a sphere of $2 \sigma$ radius in the $U V W$ space around each member. ( $\sigma$ : velocity dispersion for this association.)

and TWA, $\beta$ Pic and Tuc-Hor backwards in time. To avoid using any uncertain values for the solar peculiar motion by transforming heliocentric velocities into local standardof-rest velocities, we decided to work in a reference system centered, along time, on TWA22AB instead. We present, in Figure 2, the distance between TWA22AB and the center of each association in time. We note that TWA22AB is always closer to $\beta$ Pic than to TWA or Tuc-Hor.

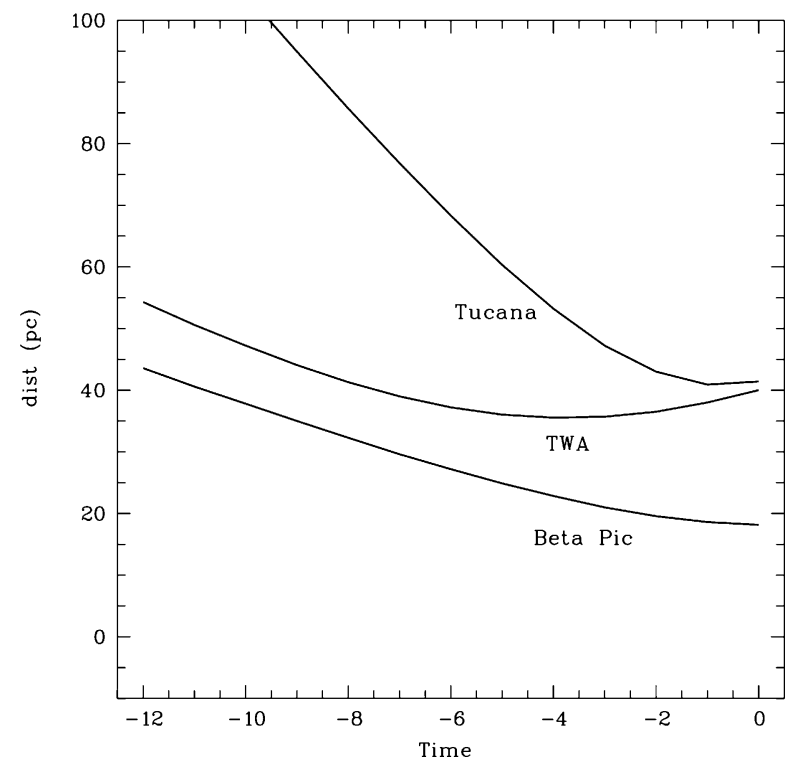

Figure 2. Distances between TWA22AB and three moving groups (TWA, $\beta$ Pic and Tuc-Hor) for the past 12 Myr. 


\section{Conclusions}

Motivated by the importance of the young, very-low-mass astrometric binary TWA $22 \mathrm{AB}$ as an important calibration point for stellar theoretical calculations, we measured

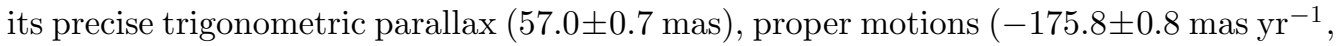

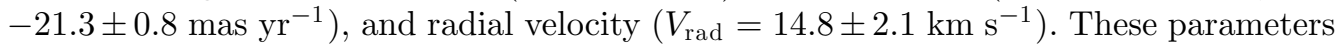
are fundamental to determine the physical properties of the tight binary system.

Our high-quality astrometric measurements along with HARPS radial-velocity measurements allow us to discuss the membership of TWA22 AB of nearby associations. Our kinematic study shows that membership of TWA22AB of known young, nearby associations can be excluded, except for the $\beta$ Pictoris and TW Hydrae associations. Membership probabilities based on the system's space motion or the use of the trace-back technique also support a possible membership of TWA22AB of the $\beta$ Pictoris association. Membership of the TW Hydrae association cannot be fully excluded due to the current lack of precise parallax measurements for most of its members.

Our results are, to some extent, inconclusive as regards the membership of TWA22AB of TWA or $\beta$ Pic, but they are consistent with that from age analysis. The location of TWA22AB in a color-magnitude diagram supports an age of about $10 \mathrm{Myr}$, but cannot be determined precisely enough to distinguish between 8 (TWA age) and $12 \mathrm{Myr}(\beta$ Pic moving group age). Precisely known trigonometric distances of many more TWA members, an aim of our larger astrometric program to observe all known TWA members, should soon improve this situation.

\section{References}

Barrado y Nevascués, D., Stauffer, J. R., Song, I., \& Caillault, J. P. 1999, ApJ (Letters), 520, L123

Bonnefoy, M., Chauvin, G., Dumas, C., Lagrange, A.-M., Beust, H., Desort, M., Texeira, R., Ducourant, C., Beuzit, J.-L., \& Song, I. 2009, A\& A, 506, 799

Ducourant, C., Teixeira, R., Krone-Martins A., Le Campion, J. F., \& Chauvin, G. 2008, RMxAC, 34,29

Kastner, J. H., Zuckerman, B., Weintraub, D. A., \& Forveille, T. 1997, Science, 277, 67

Mamajek, E. 2005,ApJ, 634, 1385

Song, I., Zuckerman, B., \& Bessel, M. S. 2003, AJ, 599, 342

Song, I., Schneider, G., Zuckerman, B., Farihi, J., Becklin, E. E., Bessell, M. S., Lowrance, P., \& Macintosh, B. A. 2006, ApJ, 652, 724

Teixeira, R., Ducourant, C., Chauvin, G., Krone-Martins, A. G. O., Bonnefoy, M., \& Song, I. 2009, A\&BA, 503, 281

Torres, C. A. O., da Silva, L., Quast, G. R., de la Reza, R., \& Jilinski, E. 2000, AJ, 120, 1410

Zuckerman, B., \& Webb, R. A. 2000, ApJ, 535, 959 